\title{
Shape Analysis and Recognition Based on Skeleton and Morphological Structure
}

\author{
Donggang Yu ${ }^{1}$, Jesse S. Jin ${ }^{1}$, Suhuai Luo ${ }^{1}$, Wei Lai ${ }^{2}$, Mira Park ${ }^{1}$ and Tuan D. Pham ${ }^{3}$ \\ ${ }^{1}$ School of Design, Communication and Information Technology, \\ University of Newcastle, NSW 2308, Australia \\ ${ }^{2}$ Faculty of Information and Communication Technologies \\ Swinburne University of Technology \\ Hawthorn, VIC3122, Australia \\ ${ }^{3}$ The University of New South Wales \\ Canberra,ACT2600,Australia
}

\begin{abstract}
This paper presents a novel and effective method of shape analysis and recognition based on skeleton and morphological structure. A series of preprocessing algorithms, smooth following and liberalization are introduced, and series of morphological structural points of image contour are extracted and merged. A series of basic shapes and a main shape of object image are described and segmented based on skeleton and morphological structure. Object shape is efficiently analyzed and recognized based on the extracted series of basic shapes and main shape. Comparing with other methods, the proposed method need not sample training set. Also, the new method can be used to analyze and recognize the shape structure of any shape, and there is no any requirement for the processed image data set. The new method can be used in image analysis, intelligent recognition, techniques, applications, systems and tools.
\end{abstract}

Key Words:

Shape analysis and recognition, morphological structure, skeleton, basic shape, main shape.

\section{Introduction}

Shape analysis and recognition plays an important role in image analysis, intelligent recognition, techniques, applications, systems and tools, and they are a difficult task. Many methods and algorithms have been developed [1, 2, 3, 4, 5, 6, 7]. The main drawbacks of the above methods are they cannot simulate the analysis and recognition method used by human in which a shape is decomposed as some basic shapes. These descriptions cannot form series of sets, or the inner contour of a binary image can not be processed based on these algorithms [6, 7], which make the analysis and understanding of contour shape difficult.

In this paper, a novel method of shape analysis and recognition is developed based on skeleton and morphology structure. Some preprocessing algorithms such as smooth following, linearization and morphological structural (MS) points are introduced in Section 2. Shape analysis and recognition (basic and main shape) are described in Section 3. Finally, a conclusion is given in Section 4. The diagram of processing is shown in Fig. 1 .

\section{Preprocessing}

Let the starting point of an binary image be the upper-left corner. Freeman code is used, and the contours are 8-connected.

\section{Contour smooth following}

Let the starting point of an binary image be the upperleft corner. The chain code set of its contour $k$ is represented as:

$$
C_{k}=\left\{c_{0}, c_{1} \ldots c_{i}, \ldots c_{n-1}, c_{n}\right\}
$$




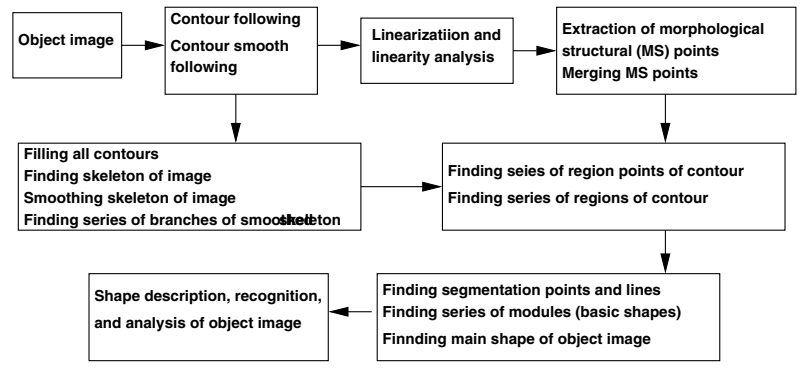

Figure 1. The diagram of object shape analysis and recognition.

where $C_{k}$ is the chain code set of contour $k$, and $i$ is the index of the contour pixels. The chain code is eightneighboring Freeman code. The difference code is defined as:

$$
d_{i}=c_{i+1}-c_{i} .
$$

In the smoothed contour, $\left|d_{i}\right|$ equals 0 or 1 [8]. One

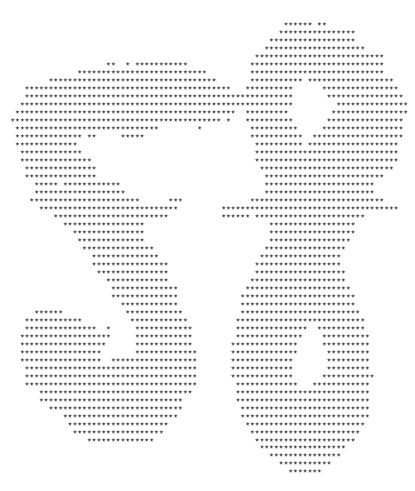

(1)

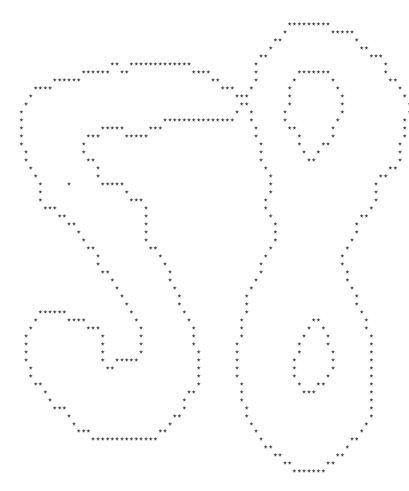

(2)
Figure 2. (1) One original binary handwritten digit image; (2) Smooth following result.

original binary handwritten digit image is shown in Fig. 2(1), and its smooth following result is shown in Fig. 2(2).

\section{linearization of smooth followed contour}

The smoothed contour can be converted to a set of lines, which consist of ordered pixels. Our task here is to approximate the contour of an image using straight lines. We call this procedure linearization of a contour.

Suppose that the direction chain code set of linearized line is

$$
\left\{c_{l}^{l n}[i] \quad\left(i=0, \ldots\left(n_{l}^{l n}-1\right)\right)\right\},
$$

where $l n$ is the $l n$-th line of a smoothed contour and $n_{l}^{l n}$ is the number of points of the $l n$-th line [8], then

$d_{i j}=c_{l}^{l n}[i]-c_{l}^{l n}[j] \quad(i=0, \ldots k-1),(j=0, \ldots k-1)$,

and

$$
\left|d_{i j}\right| \leq 1 \quad(i=0, \ldots k-1),(j=0, \ldots k-1) .
$$

The linearity of a linearized line is the variance measure between a linearized line and the linked straight line (representing with $l_{s e}$ ) between the starting and end points of the linearized line. The convexity or concavity of a linearized line describe the global variance trend of the linearized line along a direction of chain code. If linearity of a linearized line is minimum, 0 , then the line is the same as its $l_{s e}$, and it is neither convex nor concave. If linearity of a linearized line is largest, the linearized line has maximum convexity or concavityand it should be decomposed as two linearized lines.

\section{MS points}

The definition of different MS points is based on pattern models shown in Fig. 3. There are two types of MS points. One type is that the MS points are extracted based on the neighboring linearized lines. For example, the convex MS point in the direction of code 4 is represented with the character " $\mathrm{M}$ " corresponding number 4 and the concave MS point with the character " $m$ " corresponding number 24. Another type is that there are one or two MS points in a linearized line if the linearized line linearity value is large. For example, the convex MS point in the direction of code 5 is represented with the character "E" corresponding number 5 and the concave MS point with the character " $\mathrm{e}$ " corresponding number 25 .

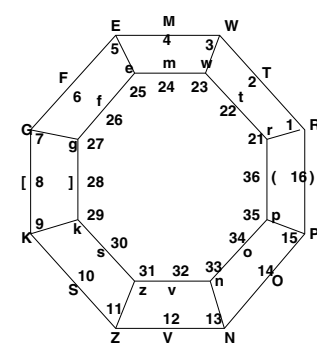

Figure 3 . The character and number pre-
sentation of thirty two MS points.

\section{Analysis and recognition of object shapes}

\section{(A) Basic shape}

Basic shapes are defined and described as follows. 
Series set of MS points of contour:

Series set of MS points of a contour can be found from the starting point of the contour in anticlockwise representing as

$$
\left\{l s[i][x, y, p, n] \quad\left(i=0, \ldots n_{s-1}\right)\right\},
$$

where $n_{s}$ is the number of MS points, $x, y, p$ and $n$ are $x, y$ coordinates, MS property, and order number in the contour for $i$-th MS point of the contour respectively.

\section{Basic polygon shape}

Basic polygon can be defined as follows: if there is no concave MS point in a contour, then the contour shape is a polygon.

Three sample images are shown in Fig. 4 and their se-

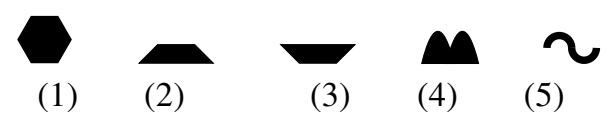

Figure 4. Five examples of basic shapes.

ries of MS points can be extracted and shown in Fig. 5. Furthermore, if successive convex or concave MS

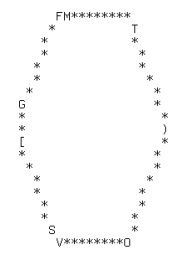

(1)

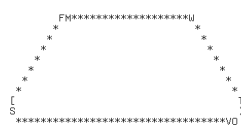

(2)

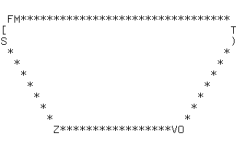

(3)
Figure 5. Series of MS points of three polygon shapes.

points are closest, there is a vertex point in the contour. The distance threshold between two MS points is 2.4 points, three MS points 3.1 and four MS points 3.6. If two MS points, $l s[i][x, y, p, n]$ and $l s[i+1][x, y, p, n]$ are closest, then MS point, $l s[i][x, y, p, n]$ is removed and the code value, $l s[i+1][p]$ is found as follows:

$$
l s[i+1][p]=\frac{(l s[i][p]+l s[i+1][p])}{2}(\bmod )
$$

where mod 16 for convex MS points, and mod 36 for concave MS points. Merging processing results of samples in Fig. 5 are shown in Fig. 6.

Triangle: If there are three merging convex MS points and there is no concave MS point in a contour, then the contour shape is recognized as a triangle.

Quadrangle: If there are four merging convex MS points in a polygon, then it is recognized as a quadrangle.

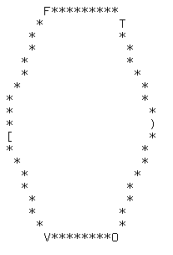

(1)

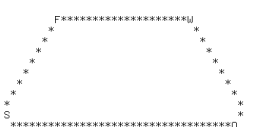

(2)

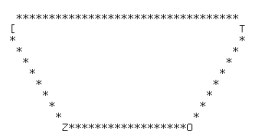

(3)

\section{Figure 6. Series of merging MS points of three polygon shapes.}

Hexagon: If there are six merging convex MS points in a polygon, then it is recognized as a hexagon.

Let $c l_{t}$ be the total length of a contour and $l_{i, i+1}, i=$ $0, \ldots, n-1$ be the length between $i$-th and $[i+1]$-th merging MS points, where $n$ is the number of merging MS points, then the series of length rate

$$
l_{r}[i, i+1]=\frac{l_{i, i+1}}{c l_{t}}
$$

can be found. Let be $l m_{i, i+1}^{d}, i=0, \ldots, n-1$ be the code value difference between $i$-th and $[i+1]$-th merging MS points, where $n$ is the number of merging MS points, then the series of code value difference can be found as follows:

$$
\operatorname{lm}_{i, i+1}^{d}=\operatorname{lm}[i+1][p]-\operatorname{lm}[i][p]
$$

\section{The size invariance of shape patterns}

The size invariance of shape patterns can be found based on the above features. For the same shape, if size (contour length) is changed, the series of length rates, the series of merging MS points, the series of merging code values and series of code value differences of the shapes are same.

\section{The rotation invariance of shape patterns}

If an shape is rotated, the rotated shapes have following rotation invariance: (a) they contain same components in their series of length rates; (b) they contain same components in their series of code value differences.

For example, two echelons in Fig. 6 have different series of merging code values, ("F":6, "S":10, "O":14 and "W":3) and ("[":8, "Z":11, "O":14 and "T":2), and they are a quadrangle because of their having four merging MS points respectively. Based on their series of code, they are a echelon which has two beveled edges with two different directions of chain code, one echelon being converted from another echelon by a rotation with 180 degree. For their rotation invariance, they have similar series of length rates and code value differences.

Half-wave: If there is one group of concave MS points 
in a shape contour, then it is a half wave.

General wave: If there are more than one group of concave MS points in a shape contour and there is no "junction" point in its skeleton, then it is a general wave. Two example shapes are shown in Figs. 4(4-5).

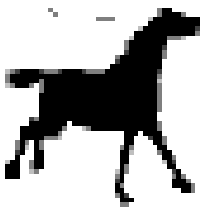

(1)

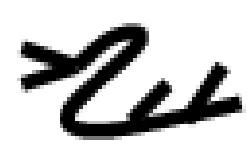

(2)

\section{Figure 7. (1) Sample image 1; (2) Sample} image 2.

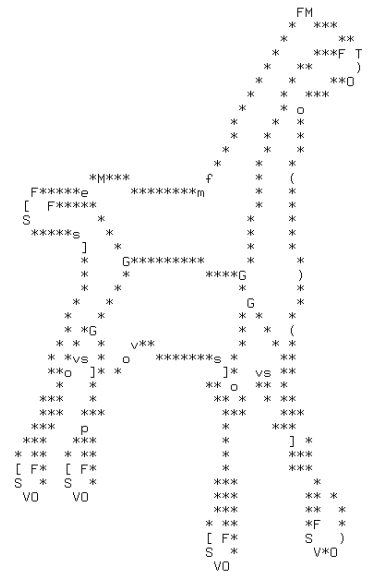

(1) Sample 1.

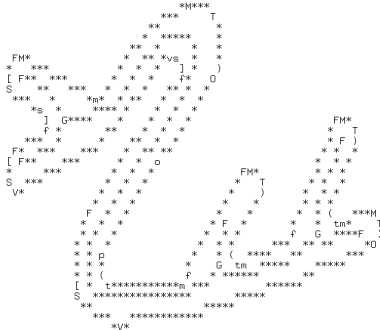

(2) Sample 2.
Figure 8. The MS points, skeleton, region points and regions of two sample shapes (samples 1 and 2).

\section{(B) Analysis and recognition of object shapes}

An object shape can be decomposed to series of basic shape modules from the starting point of shape contour in anticlockwise if it is not a basic shape. The algorithm can be described as follows: (1) determine series of region points on image contour based on the skeleton of the object image skeleton; (2) between the neighboring region points, preselect the segmentation points that are the concave MS point between which and its corresponding skeleton component there is minimum distance; (3) correct the preselected segmentation points based on the comparison result of two minimum distances, the minimum distance found in Step (2) and the minimum distance between the contour point of the re-

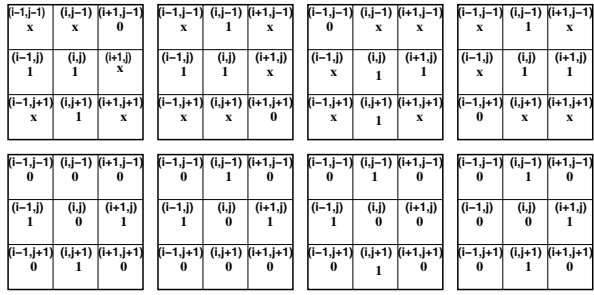

(1) The models for determining and deleting corner points.

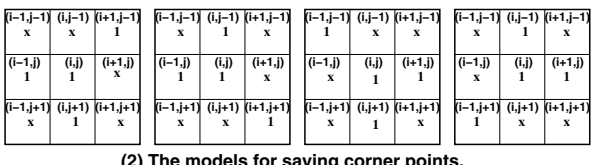

(2) The models for saving corner points.

\section{Figure 9. The patterns of smoothing skeleton.}

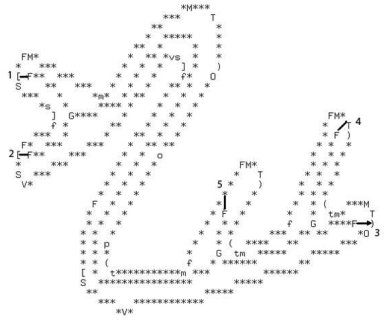

(1)
(2)

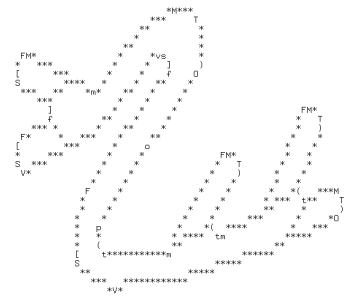

\section{Figure 10. (1) Finding region points and regions; (2) The correction of the prese- lected segmentation points and lines.}

gion and the junction point of its corresponding skeleton.

The skeletons of two sample shapes are extracted [9] and they are smoothed and shown in Figs. 8 based on the patterns in Fig. 9 where " 0 " represents background pixel, "1" represents a skeleton pixel and " $x$ " stands for "don't care" point. The region point is the contour point with minimum distance between contour point and skeleton extension point from branch "end" point in the reverse direction of the chain code of the "end" point. The contour region is between two neighboring region points. Series of region points and regions can be found. One example is shown in Fig. 10(1) where there are five region points and regions. The preselected segmentation point is a MS point of a region between which and its neighboring skeleton branch there is a minimum distance. Find one contour point of a region between which and the " $\mathrm{j}$ " point of a skeleton branch there is minimum distance. If this distance is less than that of the corresponding preselected segmentation point, then the found contour point is a segmentation 


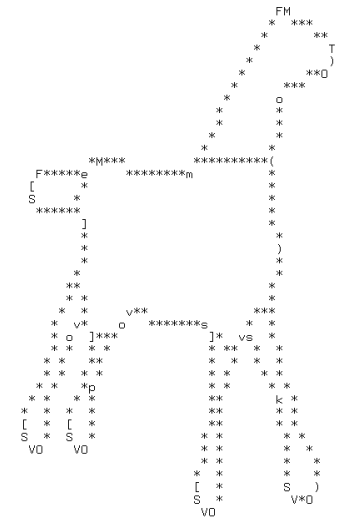

(1) Sample 1.

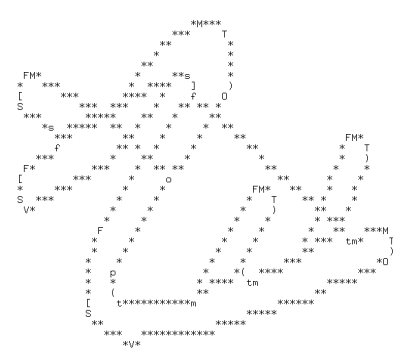

(2) Sample 2.
Figure 11. The preselected segmentation points and lines of object shapes in Fig. 7.

point in stead of the preselected segmentation point. Otherwise the preselected segmentation point is a segmentation point. Two segmentation points and lines in Fig. 11(2) are corrected and shown in Fig. 10(2) based on the above algorithm. Two neighboring selected segmentation points with a same skeleton branch construct a pair of segmentation points to form one segmentation line. Series of segmentation lines of two examples can be found and shown in Figs. 11(1) and 10(2). Series of segmented modules and main shape of the object shapes in Figs. 11(1) and 10(2) can be found and shown in Figs. 12 and 13 based on the contour points between neighboring segmentation points and segmentation lines. Basically, these segmented modules and main shape belong the patterns of basic shape. For example, the series of modules in Fig. 12(1) is that: (1) tail; (2) hind legs; (3) front legs; (4) head, and the main shape in Fig. 13(1) is the body of the horse. Each part can be analyzed and recognized based on the patterns of basic shape.

If an image shape contains inner contours, process all filled contour shapes (both the outer contour and all inner contours in the image shape) based on the proposed algorithms which are used to process the shapes with no inner contour, and the image shape can be analyzed and recognized. One sample shape is shown in Fig. 14(1), which is the extracted binary images of lily flowers which have six petals. Its smooth followed outer and inner contours, MS points, segmentation lines and main shapes are fond and shown in Figs. 14(2,3) based on the above algorithms. The outer shape of the sample shape 3 is recognized as a six-pointed star based on its series of modules (see Fig. 15(1)) of outer con-
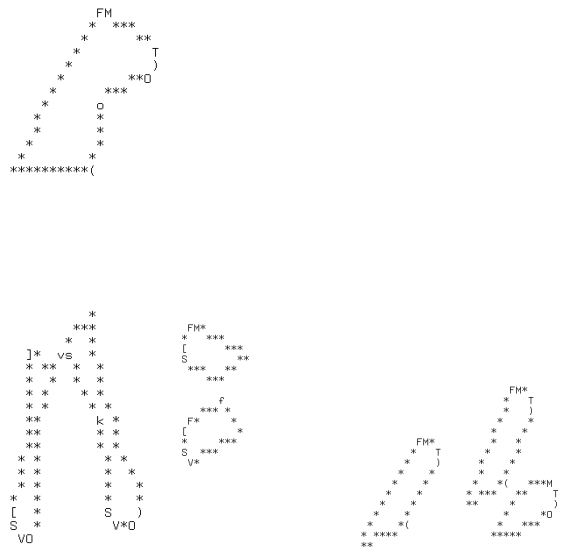

(1) Sample 1.

(2) Sample 2.

\section{Figure 12. Series of segmented modules of two sample shapes.}

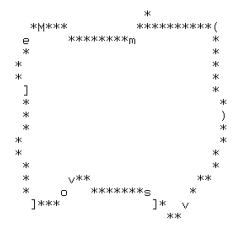

(1) Sample 1.

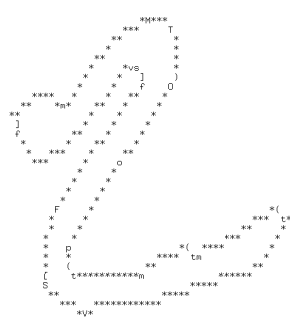

(2) Sample 2.

\section{Figure 13. Main shape of two sample shapes.}

tour and its main shape (see the large in Fig. 14(3)) which is a hexagon based on the definition of basic shape. The shape of the flower center is described, recognized and analyzed in terms of its series of modules (see Fig. 15(2)) of inner contour and inner contours's main shape (see the small shape in Fig. 14(3)). Here the prior knowledge is used to recognize them as lily flower since the prior knowledge demonstrates that the object image belong flower. Possibly they could be recognized as a Indo-Pacific star (starfish) in marine environment or a star in a painting etc.

\section{Conclusion}

An efficient and new method has been developed to analyze and recognize the image shape. The object shapes are segmented as a series of modules and main shape (basic shapes) based on MS points of contour regions, skeleton structure and contour point distribution of contour regions, which can be used to 


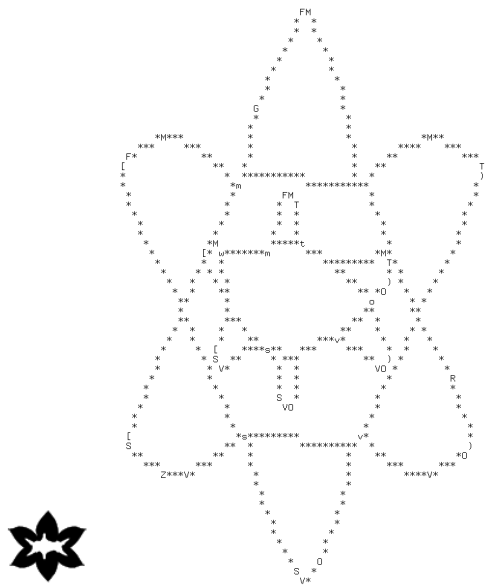

(1)

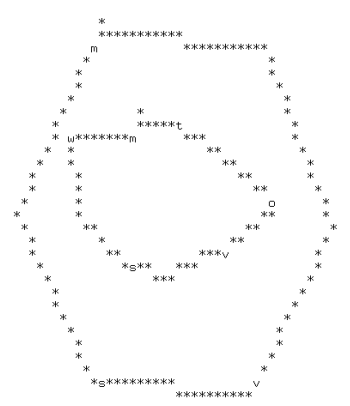

(3)
Figure 14. (1) Sample shape 3; (2) Segmentation lines; (3)Main shapes of sample shape 3

described, analyze and recognize object shapes. The best useful contribution is that some novel algorithms and concepts such as basic shape, main shape, series of modules, smooth following of contour and skeleton etc. are first proposed and described in the paper, but other methods not [7, 6]. For some complicated images such as sample shape 3, other shape analysis and recognition methods cannot be used to describe it well. Particularly, if a object is rotated, the related rotation information can be found and it is important to describe the position change of the object image. Comparing with other methods, the proposed method need not sample training set. Also, the new method can be used to analyze and recognize the shape structure of any shape, and there is no any requirement for the processed image data set.

Acknowledgement- The project is partially supported by ARC Discovery Grant DP0773584 and ARC LP0347156.

\section{References}

[1] F. Moktarian and A. K. Mackworth, A Theory of Multiscale Curvature-Based Shape Representation for Planer Curvature Angles, IEEE Trans. Pattern Analysis Mach. Intell., 14 , (8), pp. 789-805, 1992.

[2] M. Sonka, V. Hlavac and R. Boyle, Image Processing, Analysis and Machine Vision, Chapman \& Hall Computing, Cambridge, 1993.

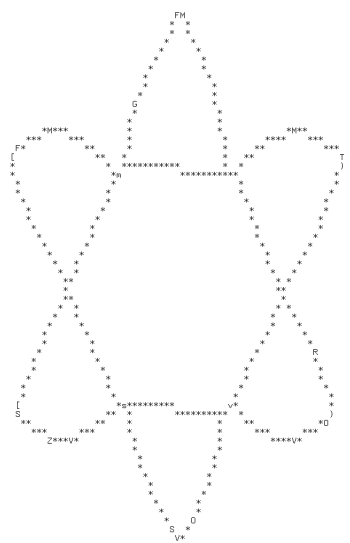

(1)

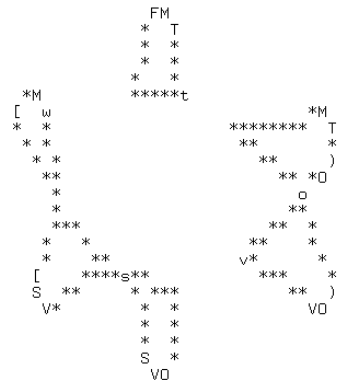

(2)
Figure 15. (1) Series of segmented modules of outer contour of sample shape 3; (2) Series of segmented modules of inner contour of sample shape 3 .
[3] N. Ueda and S. Suzuki, Learning Models from Shape Contour Using Multiscale Convex/Concave Structure Matching, IEEE Trans. Pattern Analysis Mach. Intell.,15 , (4), pp. 337-352, 1993.

[4] N. Katzir, M. Lindenbaum and M. Porat, Curvature Angle Segmentation under Partial occlusion, IEEE Trans. Pattern Analysis Mach. Intell.,c 16, (5), pp. 513-519, 1994.

[5] A. M. N. Fu, H. Yan, K. Huang, A Curvature Angle Bend Function Based Method to Characterize Contour Shapes, Patt. Recog., 30 , 1661-1671, 1997.

[6] S. Berretti ect., Retrieval by shape similarity with perceptual distance and e ective indexing, IEEE Trans. Multimedia 2, pp. 225-239, 2000.

[7] D. Zhang and G. Lu, Review of shape representation andd escription techniques, Pattern Recognition, 37, pp. 1-19, 2004.

[8] D. Yu and H. Yan, An efficient algorithm for smoothing, linearization and detection of structure feature points of binary image contours, Patt. Recog., 30, (1), pp. 57-69, 1997.

[9] T.Y. Zhang and C.Y.Suen, "A fast parallel algorithm for thinning digital patterns," Communications of ACM, 27, pp. 236-239, 1984. 